\title{
The eukaryotic translation initiation regulator CDC123 defines a divergent clade of ATP-grasp enzymes with a predicted role in novel protein modifications
}

\author{
A Maxwell Burroughs, Dapeng Zhang and L Aravind*
}

\begin{abstract}
Deciphering the origin of uniquely eukaryotic features of sub-cellular systems, such as the translation apparatus, is critical in reconstructing eukaryogenesis. One such feature is the highly conserved, but poorly understood, eukaryotic protein CDC123, which regulates the abundance of the eukaryotic translation initiation elF2 complex and binds one of its components elF2y. We show that the eukaryotic protein CDC123 defines a novel clade of ATP-grasp enzymes distinguished from all other members of the superfamily by a RAGNYA domain with two conserved lysines (henceforth the R2K clade). Combining the available biochemical and genetic data on CDC123 with the inferred enzymatic function, we propose that the eukaryotic CDC123 proteins are likely to function as ATP-dependent protein-peptide ligases which modify proteins by ribosome-independent addition of an oligopeptide tag. We also show that the CDC123 family emerged first in bacteria where it appears to have diversified along with the two other families of the R2K clade. The bacterial CDC123 family members are of two distinct types, one found as part of type VI secretion systems which deliver polymorphic toxins and the other functioning as potential effectors delivered to amoeboid eukaryotic hosts. Representatives of the latter type have also been independently transferred to phylogenetically unrelated amoeboid eukaryotes and their nucleo-cytoplasmic large DNA viruses. Similarly, the two other prokaryotic R2K clade families are also proposed to participate in biological conflicts between bacteriophages and their hosts. These findings add further evidence to the recently proposed hypothesis that the horizontal transfer of enzymatic effectors from the bacterial endosymbionts of the stem eukaryotes played a fundamental role in the emergence of the characteristically eukaryotic regulatory systems and sub-cellular structures.
\end{abstract}

Reviewers: This article was reviewed by Michael Galperin and Sandor Pongor.

\section{Findings}

The origin of eukaryotes was marked by the emergence of entirely new subcellular systems as well as novel components in preexisting systems. Deciphering the evolutionary history and the ultimate provenance of these systems and components, which were long considered to be quintessential features of eukaryotes, has considerably advanced in the past decade as a result of the growing genomic data and concomitant comparative genomics analysis [1-4]. In this regard, we have had a long-standing interest in understanding the origins of eukaryotic innovations relating to ribosome biogenesis and the translation

\footnotetext{
* Correspondence: aravind@mail.nih.gov
National Center for Biotechnology Information, National Library of Medicine,

* Correspondence: aravind@mail.nih.gov
National Center for Biotechnology Information, National Library of Medicine, Bethesda, MD 20894, USA
} () Biomed Central

machinery [5,6]. In several cases, we have been able to identify prokaryotic homologs of what previously seemed to be purely eukaryote-specific components in these systems. Recognition of these prokaryotic versions has helped clarify the precise evolutionary trajectories by which these components were recruited to the eukaryotic ribosome biogenesis/translation apparatus. Moreover, these studies have also often helped predict the potential biochemical roles of several poorly understood components in these systems by exploiting the contextual information available in prokaryotic genomes $[5,6]$.

In this study we present an investigation of the conserved eukaryotic regulator of translation initiation $\mathrm{CDC} 123$ and it homologs. CDC123 was first identified over 30 years ago in a screen for temperature-sensitive mutations that blocked cell proliferation in rat fibroblast cells [7]. 
This was attributed to a cell-cycle related function arising from its apparent functional interaction with checkpoint proteins chf1/chf2 [8], which are active in triggering mitosis entry [9]. Conditional mutants in the Saccharomyces cerevisiae cognate were shown to result in increased heat sensitivity, whereas $\mathrm{CDC} 123$ null mutants were inviable [8]. Further investigation of these phenotypes pointed to a role in translation as it was observed that $\mathrm{CDC} 123$ specifically regulates the abundance of the eukaryotic translation initiation eIF2 complex [8,10], and binds one of its components yeast GCD11 or its human ortholog eIF2 $\gamma$ $[11,12]$ in the cytoplasm. To date its orthologs have only been reported from eukaryotes, where it is widely distributed across all major lineages of the eukaryotic tree. This phyletic pattern, together with its essentiality in yeast, suggest that $\mathrm{CDC} 123$ might indeed be a conserved regulator of translation. However, despite over three decades of research on $\mathrm{CDC123}$, its precise role in translation or cell-cycle regulation remains unclear. Given these observations and the mounting evidence suggesting possible links between CDC123 and a variety of human disease states including breast cancer [13], type II diabetes [14], and COPD [15], we sought to apply state-of-the-art methods in comparative sequence and genome analysis to better understand the biochemical roles of CDC123.

We show that CDC123 defines a novel, highly-derived clade of the ATP-grasp superfamily of enzymes $[16,17]$. We define the conserved sequence and structure features of this clade of ATP-grasp proteins and predict they are likely to catalyze protein modifications by the formation of amide/peptide-like linkages in an ATP-dependent manner. In addition, we identify the first bacterial homologs of CDC123 where they are often found as part of the type VI secretion systems (T6SS) that deliver polymorphic toxins $[18,19]$. Further, we show that this clade of ATP-grasp domains additionally includes two previously unknown, related prokaryotic families with potential roles in distinct biological conflict systems [20-22]. Finally, we present evidence that the eukaryotic CDC123s might have been derived from an ancestral bacterial conflict system in the stem eukaryote and recruited for a role in protein modifications, including in context of translation initiation.

\section{CDC123 contains an ATP-grasp module and has several distinct bacterial homologs}

To better characterize CDC123, we initiated iterative sequence profile searches with CDC123 orthologs known from prior studies as queries using the PSI-BLAST and JACKHMMER programs (see Methods). Beyond the previously-identified homologs in animals, plants, fungi, and stramenopiles [8], we detected eukaryotic orthologs spanning all other major branches of the eukaryotic tree. For example, a search initiated with the yeast $\mathrm{CDC} 123$ recovered orthologs from the apicomplexans, kinetoplastids, parabasalids and diplomonads within 2 iterations with PSI-BLAST (Additional file 1). Concomitantly, these searches also recovered sequences from diverse bacterial and viral lineages. For example, the above search recovered sequences from the $\gamma$-proteobacteria Erwinia chrysanthemi (gi: 654084322, iteration: 1; e-value 6e-6) and Legionella pneumophila (gi: 652968979; iteration: 2; e-value: 2e-08), the planctomycete Zavarzinella formosa (gi: 521962559, iteration: 2; e-value: 8e-09), and the nucleocytoplasmic large DNA virus (NCLDV) [23] Megavirus Iba (gi: 448825053; iteration: 2; e-value 1e-11).

Reverse searches initiated with these bacterial sequences recovered their eukaryotic counterparts in initial iterations, then recovered several prokaryotic sequences either unannotated or annotated as containing the "Domain of Unknown Function", DUF4343 [24], before finally recovering sequences containing known ATP-grasp domains, typically those most-closely related to RimK and RimK-like ATP-grasp families [16]. For example, a search initiated with the bacterial CDC123 homologue from Lentisphaera araneosa (gi: 494490064) recovers a sequence annotated as containing the DUF4343 domain from bacterium Deinococcus pimensis (gi:653301678; iteration: 4; e-value: 4e-3), a sequence from the bacterium Pseudomonas aeruginosa with no previously identified domain (gi: 489255144; iteration: 6; e-value: 4e-05), and a RimKlike ATP-grasp fold [25] domain from Herpetosiphon aurantiacus (gi: 501142781; iteration: 8; e-value: 2e-04). We further confirmed these results using an HMM-(Hidden Markov Model) based method for detecting distant homology. HMMs constructed with the $\mathrm{CDC} 123$ sequences as seeds were searched against a library of pre-constructed HMMs based on Pfam domain definitions [26] and solved PDB (Protein Data Bank [27]) structures with the HHpred program. The strongest relationship detected in these searches was consistently with the Pfam DUF4343 domain, followed by detection of other ATP-grasp families and structures including the Pfam DUF3182 domain, a heretofore unrecognized member of the ATP-grasp fold sharing conserved features and general sequence affinity with a clade of ATP-grasp enzymes including the carbamoyl phosphate synthases and Btr--like butirosin biosynthesis enzymes (Additional File 1). For example, a HMM constructed using the yeast $\mathrm{CDC} 123$ sequence as a seed detected a significant relationship with the DUF4343 Pfam domain (p-value: 5.7e-10), the RimK-like ATP-grasp domain (p-value: 9.8e-07), and the RimK structure from Thermus thermophilus (PDB: 3VPD; $\mathrm{p}$-value: 6.7E-06). However, in terms of reciprocal recovery in sequencesimilarity searches and sequence similarity- and lengthbased clustering with the BLASTCLUST program (see Methods), none of CDC123 and its newly identified homologs overlapped with any previously known ATP-grasp families $[28,16]$. Together, these results strongly suggest 
that these sequences define a previously-unrecognized clade of ATP-grasp-like proteins, which includes the CDC123, DUF4343-containing proteins, and several additional unannotated prokaryotic proteins.

\section{Distinctive features of the novel ATP-grasp clade and identification of three distinct families within it}

The catalytic module of the ATP-grasp superfamily is constructed from two distinct domains: the N-terminal RAGNYA domain and the C-terminal protein kinase/PIPKlike domain [29-31]. In addition to this catalytic module, most members of the ATP-grasp superfamily are fused at the $\mathrm{N}$-terminus to the pre-ATP-grasp domain [16]. The position of the catalytic residues are typically conserved across the superfamily and include: 1) a positively-charged residue, typically a lysine, found in the linker region connecting the pre-ATP-grasp domain with the RAGNYA domain, 2) an additional positively-charged residue, again typically a lysine, found near the C-terminal end of the second strand of the RAGYNA domain, 3) an acidic residue, typically an aspartate, located in the central region of the fourth strand of the protein kinase-like domain, and 4) a conserved motif typically of the form ExN (where ' $\mathrm{x}$ ' is any residue) located at the C-terminus of the fifth and final conserved strand of the protein kinase domain [16]. Additionally, a large, monophyletic clade of ATP-grasp superfamilies, including most peptide/amide bond-forming ligases members, contain a conserved arginine residue in the first strand of the protein kinase-like domain [16] (Additional File 1).

Comparison of the features of the newly-identified clade to the above-described ATP-grasp template revealed considerable concordance (for example: K104, D233, and D246xN248 correspond to features 2-4 listed above in the human CDC123 protein). However, striking differences were observed: 1) In other ATP-grasp families the loop between strands 2 and 3 of the RAGNYA domain is wellconserved in terms of length (usually 9 amino acids) and harbors a conserved ssxGbGl motif (where 's' is any small residue, 'b' is any big residue, and ' $\mathrm{l}$ ' is any aliphatic residue) [16]. However, in this novel clade this loop displays considerable length diversity and lacks the above sequence motif. 2) The lysine typically observed in the linker region between the pre-ATP-grasp domain and the RAGNYA domain is consistently absent in all members of this newly detected clade (Figure 1). Instead, they display a distinct conserved lysine/arginine in the above-stated loop, just downstream of the absolutely conserved lysine in strand 2 (Figure 1). This loop region is spatially positioned in close proximity to the active site [28]. Hence, we predict the conserved lysine/arginine from this loop likely acts as a secondarily-acquired, compensatory residue that functions in lieu of the conserved lysine from the pre-ATPgrasp-RAGNYA linker region. Indeed, these shared features strongly support the monophyly of this clade of ATP-grasp enzymes and we propose naming this novel clade the R2K ATP-grasp clade, for RAGNYA-containing 2 lysines $(K)$.

To further understand the relationships within the R2K clade, we clustered its representatives using sequence similarity- and length-based scoring parameters with the BLASTCLUST program (Additional file 1). The results identified three distinct families: 1) the CDC123 or R2K.1 family consisting of the pan-eukaryotic CDC123like proteins, close homologs in certain NCDLVs infecting microbial eukaryotes, and bacterial versions from $\alpha-, \delta$, and $\gamma$-proteobacteria, planctomycetes, lentisphaerae, and firmicutes; 2) the R2K.2 family sporadically present across many bacteria and a few bacteriophages, typically annotated as matching the Pfam DUF4343 model; 3) the R2K.3 family with a similar phyletically wide, yet sporadic, distribution in bacteria with rare archaeal representatives. The R2K.3 family is often misannotated as a "membrane protein", typified by the sce1853 protein in Sorangium cellulosum. Each of the families is clearly distinguished from the other by the spacing of the second conserved lysine with respect to the absolutely conserved lysine in strand 2 of the RAGNYA domain (Figure 1). A subset of the families or members within each family might show certain peculiarities: the eukaryotic versions of the CDC123 family are often characterized by large, variable, low complexity inserts within the catalytic module predicted to be structurally disordered. The pre-ATP-grasp domain is well-conserved in the R2K.3 family but is rapidly diverging in the CDC123 and the R2K.2 families. The R2K.3 family is further distinguished by an unusual constellation of conserved residues in the final strand of the protein kinase/PIPK-like domain of the ATP-grasp module, where it contains an ExGD motif instead of the standard ExN motif (Figure 1). While the $\mathrm{N}$ residue is, on occasion, substituted for distinct polar residues, the migration of the residue one position downstream has not, to our knowledge, previously been observed in the ATP-grasp superfamily.

\section{Evolutionary history of the R2K clade ATP-grasp enzymes} Despite their distinctive features, the fusion to the preATP-grasp domain points to the R2K clade being deeply nested within the previously defined tree of ATP-grasplike modules [16] (Additional File 1). Moreover, the presence of the conserved arginine residue in the first strand of the protein kinase/PIPK-like domain of the ATP-grasp module (part of the conserved ExR motif in S5 of Figure 1) suggests that the R2K clade specifically belongs to a larger assemblage within the superfamily that is almost entirely comprised of ligases catalyzing peptide-like linkages [16]. This assemblage includes the ATP-grasp enzymes catalyzing the formation of such bonds in cofactors (e.g. glutathione), antibiotics [32,33], peptidoglycan [34,35], siderophores [36], the biosynthesis of lysine (LysX), and 


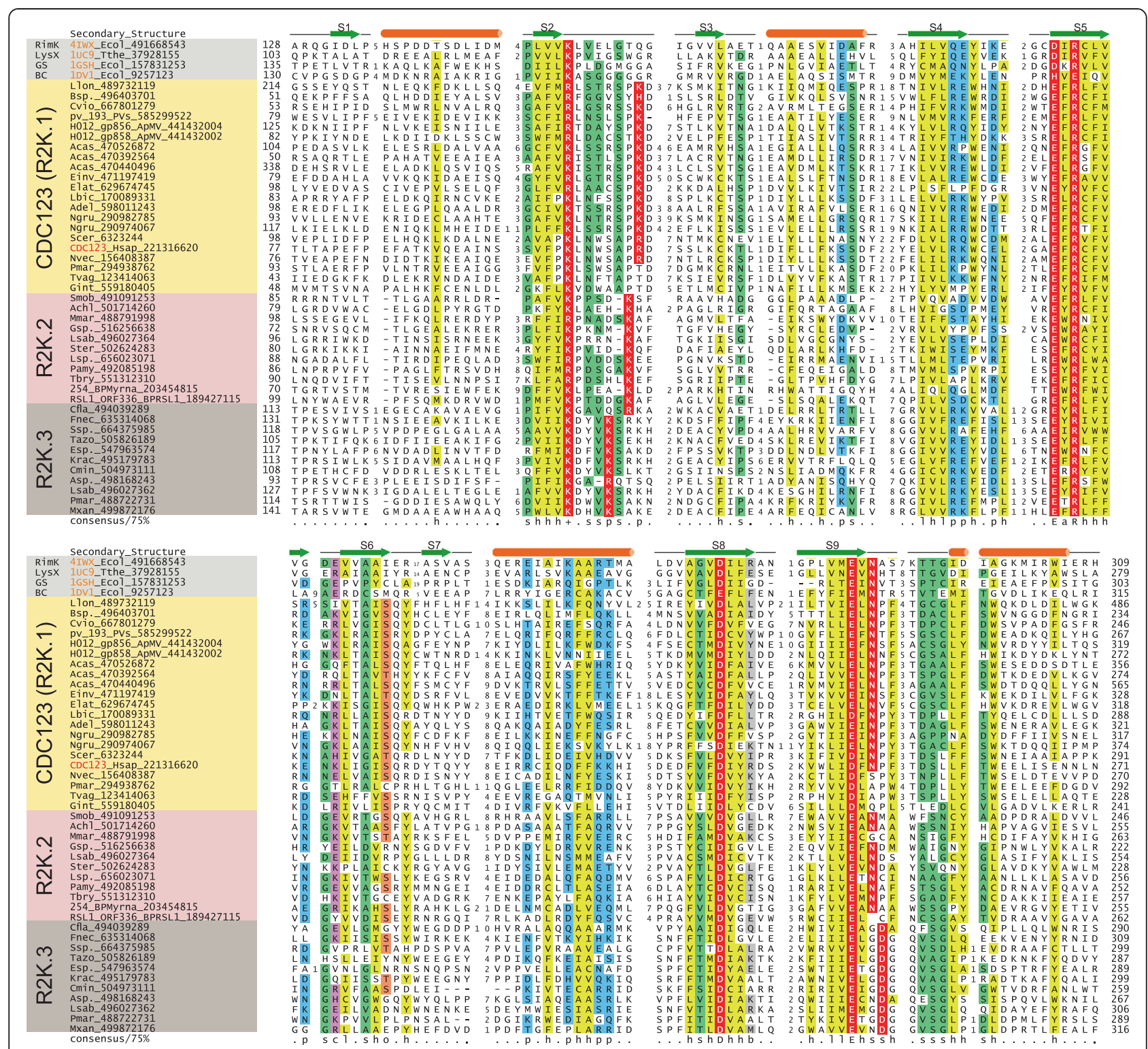

Figure 1 Multiple sequence alignment of three families of R2K ATP-grasp modules with known ATP-grasp structures. Proteins are labeled with their species abbreviations and GenBank index numbers along with gene names for human and viral homologs. PDB identifiers, colored in orange, are given in lieu of gene names where applicable. Secondary structures are depicted above alignment with loop regions shown as lines, $\beta$-strands (S1-S9) shown as green arrows and a-helices shown as orange cylinders. The coloring of the alignment is based on 75\% consensus shown below the alignment, using the following scheme: h, hydrophobic (shaded in yellow); s, small (shaded in light green); l, aliphatic (shaded in yellow); p, polar (shaded in light blue); +, positively charged; b, big (shaded in gray); a, aromatic (shaded in yellow); c, charged (shaded in purple). Predicted catalytic residues are colored in white and shaded in red. Species abbreviations: Acas, Acanthamoeba castellanii; Achl, Arthrobacter chlorophenolicus; Adel, Auricularia delicata; ApMV, Acanthamoeba polyphaga moumouvirus; Asp., Acaryochloris sp.; BPMyrna, Mycobacterium phage Myrna; BPRSL1, Ralstonia phage RSL1; Bsp., Brenneria sp.; Cfla, Chthoniobacter flavus; Cmin, Chamaesiphon minutus; Einv, Entamoeba invadens; Elat, Eutypa lata; Esp., Eggerthella sp.; Fnec, Fusobacterium necrophorum; Gint, Giardia intestinalis; Gsp., Geitlerinema sp.; Hsap, Homo sapiens; Krac, Ktedonobacter racemifer; Lbic, Laccaria bicolor; Llon, Legionella longbeachae; Lsab, Lachnoanaerobaculum saburreum; Lsp., Labrenzia sp; Mmar, Microscilla marina; Mxan, Myxococcus xanthus; Ngru, Naegleria gruberi; Nvec, Nematostella vectensis; PVs, Pithovirus sibericum; Pamy, Pseudomonas amygdali; Pmar, Perkinsus marinus; Pmar, Planctomyces maris; Scer, Saccharomyces cerevisiae; Smob, Streptomyces mobaraensis; Ssp., Streptomyces sp.; Ster, Sebaldella termitidis; Tazo, Treponema azotonutricium; Tbry, Treponema bryantii; Tvag, Trichomonas vaginalis. Other abbreviations: GS, glutathione synthase; BC, biotin carboxylase.

catalyzing polyglutamyl and polyglycinyl modification of cofactors and proteins like ribosomal protein S6 and tubulin $[37,38]$. The majority of these families appear to have first radiated in the bacteria [16]. Similarly, all three families of the $\mathrm{R} 2 \mathrm{~K}$ clade have a bacterial presence, with the eukaryotic CDC123s nested within the 
bacterial diversification of this clade in a phylogenetic tree (Figure 2). These observations suggest the R2K clade first emerged in bacteria followed by initial diversification into three distinct families. Additionally, the phyletic patterns of bacterial versions and their relationships in the phylogenetic tree (Figure 2, Additional File 1) strongly suggest horizontal gene transfer (HGT) as the key theme in their evolution.

Two distinct versions of the CDC123 (R2K.1) family are found in eukaryotes. The phyletic patterns suggest that the classical $\mathrm{CDC} 123$ orthologs, typified by relatively short average branch terminal lengths (Figure 2), were likely to have been present in the Last Eukaryotic Common Ancestor (LECA), suggesting that a HGT event from a bacterial source transferred these to the stem of the eukaryotic lineage. A second set of more rapidly-evolving CDC123 family members are found primarily in phylogenetically distant amoeboid organisms like Entamoeba, Acanthamoeba, and Naegleria, often in multiple copies (Figure 2). These group with cognates from facultative

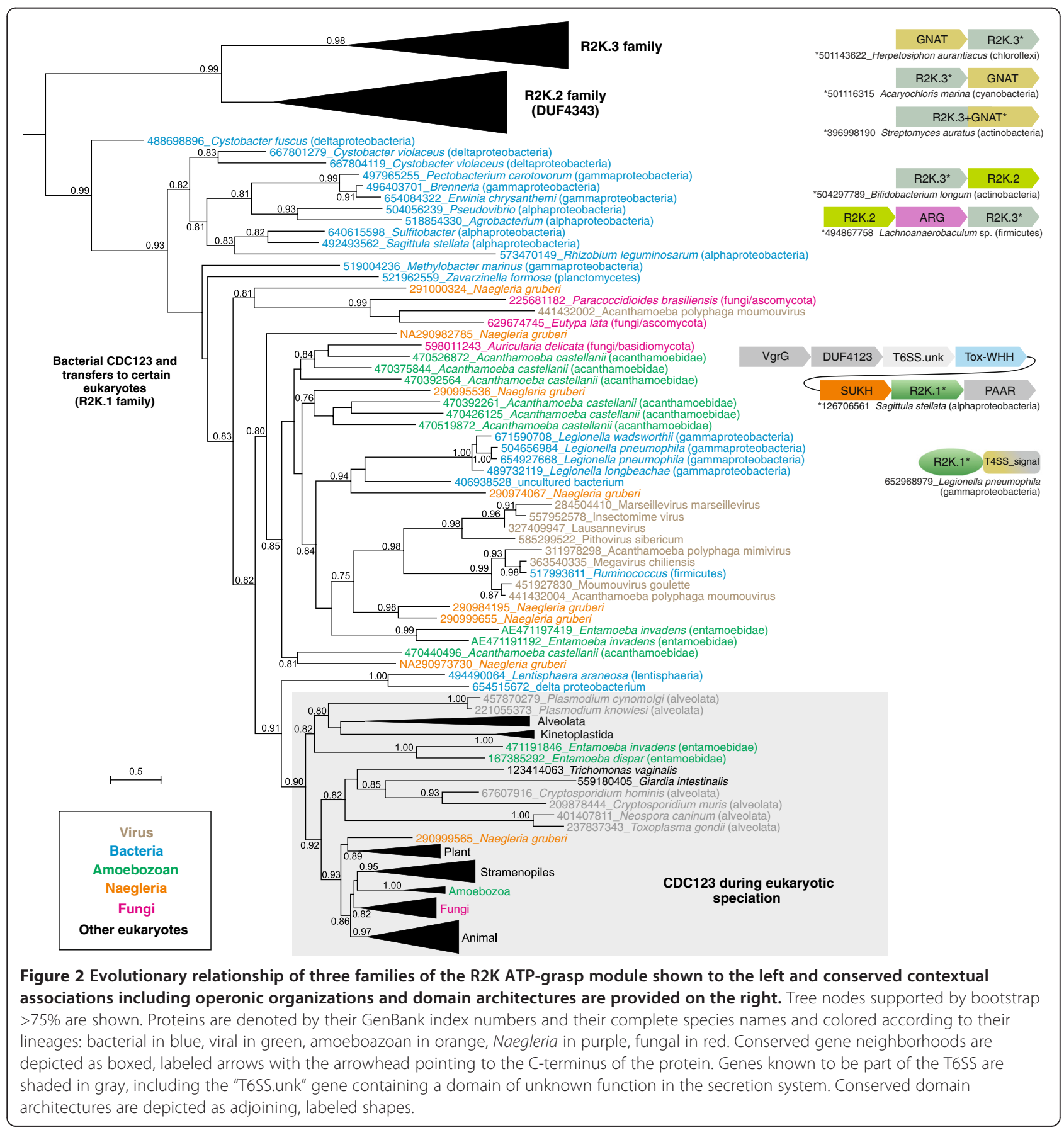


bacterial symbionts of amoebae, namely Legionella and giant NCLDVs that infect amoeboid organisms [39] (Figure 2). The complex interplay between Legionella and eukaryotic hosts $[40,41]$ has previously been proposed to have been a conduit for HGT of multiple domains $[42,43]$. Similarly, transfers between symbionts and viruses sharing the same host cell have also been documented $[44,45,39]$. Thus, the distinctive members of the R2K.1 shared by amoeboid eukaryotes and their symbionts and viruses were likely disseminated via HGT associated with these interactions.

\section{Functional inferences for R2K families based on genome contextual information and prior experimental results}

Based on the conservation of most key catalytic residues or their compensation with spatially-equivalent residues from elsewhere in the sequence we propose that most members of the R2K clade are likely to be active enzymes, although in some lineages this activity may have been lost, most notably in the eukaryotic apicomplexan clade (Figure 1, Additional File 1). Furthermore, based on the nesting of the R2K clade within the ATP-grasp assemblage, which primarily catalyzes the formation of peptide-like linkages [16] (Additional file 1), we propose that members of this clade are likely to catalyze similar reactions. Yeast strains overexpressing CDC123 displayed a second, slightly larger isoform of CDC123 at low levels [46]. This isoform was suggested to result from an unknown modification to CDC123 and was linked to its proteasomal degradation [47]. The same work ruled out ubiquitin- and phosphorylgroup additions as potential modifications resulting in this isoform [47]. In light of the peptide-bond forming activity predicted for the R2K clade ATP-grasp proteins, we posit that the observed isoform perhaps results from automodification via serial ligation of amino acids to a particular sidechain or the C-terminus comparable to the modifications catalyzed by RimK on the ribosomal protein $\mathrm{S} 6$ or the TTLs on tubulins. RimK has been shown to ligate up to fifteen glutamate residues to S6 [48,25]; auto-ligation of a comparable number of amino acid residues would be sufficient to explain the observed larger isoform of CDC123. The interaction networks for various CDC123 eukaryotic orthologs inferred from high-throughput interactome studies show an enrichment for multiple proteosomal components [49]. This, together with heat-sensitivity of the CDC123 mutants, suggests that one consequence of this modification might be to regulate the stability of proteins via the proteasome. However, it is likely that the CDC123-catalyzed modification has a distinct role in the context of translation initiation. Physical interaction of CDC123 with GCD11/eIF2 $\gamma$ and the marked decrease in eIF2 complex formation without changes in concentration of individual eIF2 complex components in the CDC123 null mutants [10] suggest that the modification of particular components might facilitate assembly of this key translation initiation complex. Similarly, the cell-cycle checkpoint proteins Chf1/Chf2 [8] might also be other targets for modification catalyzed by CDC123.

We then examined the contextual information in the form of conserved gene neighborhoods and gene fusions of the prokaryotic versions as this has proved to be a useful tool for deciphering the function of uncharacterized gene products [50,51]. Consequently, we observed that across several phylogenetically distant bacteria, genes coding for members of the CDC123 (R2K.1) family are embedded within the recently-described polymorphic toxin loci (Figure 2). Polymorphic toxin systems have been implicated in intra-specific conflicts between bacteria, acting as the arbiters of "self versus non-self" distinctions between closely-related organisms $[18,52,19,21]$. The toxin proteins from these systems are delivered to target cells via a wide range of secretory systems, which are often genomicallylinked to the core loci coding for the toxin and its cognate immunity protein [18]. Among these secretory systems is T6SS, which utilizes caudate bacteriophage tail-derived components to inject toxins into target cells [53]. We observed that $\mathrm{CDC} 123$ occurs specifically in polymorphic toxin loci with genes coding for the SUKH domain immunity protein [19] and diagnostic components of the T6SS system including the VgtG, Hcp1, and proteins with PAAR motifs [18] (Figure 2). As only a subset of polymorphic toxins delivered by T6SS encode a CDC123-like protein, it is likely to function in a supplementary role, perhaps as a secondary toxin injected into the target organism or as an auxiliary protein that regulates either the toxin, the immunity protein, or the secretory apparatus.

The CDC123 family protein found in Legionellae contains extended $\mathrm{C}$-terminal and $\mathrm{N}$-terminal regions not observed in other CDC123-like proteins (Figure 2, Additional file 1). Legionella secretes several toxins/ effectors into its eukaryotic host cell using the Type IV secretion system (T4SS). The C-terminal region of CDC123 from Legionellae harbors several of the characteristics known to be important for T4SS delivery such as: 1) a largely unstructured C-terminal region [54], 2) a conserved hydrophobic residue very close to the C-terminus [54], and 3) a preponderance of both small and polar residues in the $\sim 15$ residues upstream of the hydrophobic residue [55] (Additional file 1). Hence, it is conceivable the Legionella CDC123 is secreted via the T4SS as an effector into the host eukaryotic cell. Thus, the evidence from the two distinct sets of bacterial members of the CDC123 family point in the direction of functioning as a secreted toxin or auxiliary factors of toxin systems, which might modify proteins with peptide tags by means of their peptide ligase activity. Given the second set of eukaryotic and NCLDV CDC123 homologs are specifically related to the Legionella versions, it is likely that 
these perform functions similar to the former and different from the classical CDC123 translation regulators referred to above. Their presence, often as multiple paralogous copies (unlike the single-copy classical CDC123 versions) across phylogenetically distant amoeboid eukaryotes (Additional File 1), raises the possibility that they modify cytoskeletal proteins associated with the amoeboid cellular morphology, such as components of the actinbased cytoskeleton. This might parallel the extensive modification of tubulin by peptide tags, ranging from a single tyrosine to long polyglutamyl or polyglycinyl chains, catalyzed by multiple ATP-grasp ligases $[37,38,56,57]$. Thus, such cytoskeletal modifications could be utilized by both the amoeboid organisms and their symbionts/ parasites in facilitating formation of intracellular structures conducive to their lifestyle.

We observed operonic connections between genes of the R2K.3 family and those coding for multiple GCN5like acetyltransferase (GNAT) domains in several actinobacteria of the Streptomyces lineage, the chloroflexi Herpetosiphon, and the cyanobacterium Acaryochloris (Figure 2). In certain firmicutes and the actinobacteria, genes for the R2K.3 and R2K.2 families were linked together in the same operon (Figure 2). The operonic linkage of genes for distinct ATP-grasp peptide ligases or unrelated ligase domains, such as those of the $\mathrm{COOH}$ $\mathrm{NH} 2$ ligase or GNAT superfamilies, have been previously observed in multiple instances [16]. Such linked peptide ligases often catalyze successive peptide ligations with distinct moieties in the biosynthesis of peptide-derived secondary metabolites like antibiotics and siderophores, storage polypeptides like cyanophycin, peptidoglycan, teichuronopeptides, the $\mathrm{O}$-antigen, and cofactors like glutathione $[58,25]$. Hence, we posit that the R2K.2 and R2K.3 families catalyze peptide ligation, which might be further followed by action of the second ligase or capped by an acyl group added by the associated GNAT protein. In certain firmicutes, the linked genes for the R2K.2 and R2K.3 family proteins sandwich a third gene coding for an ADP-ribosylglycohydrolase (ARG) (Figure 2). ARGs catalyze the hydrolysis of glycosidic bonds to remove ADP-ribose moieties conjugated to side-chains of particular residues in proteins by ADP-ribosyltranferases $[59,60]$. This linkage suggests that, like the ARG, the peptide ligase action of R2K.2 and R2K.3 enzymes is likely to target proteins. As there are no other linked genes in these neighborhoods, the identity of their target proteins remain elusive. Nevertheless, given that at least the R2K.2 family is found in several caudate bacteriophages infecting phylogenetically-distant bacteria (Additional File 1), it might modify specific host proteins, analogous to the ADP-ribose modification of the same by phage enzymes [61-63]. Conversely, even as phage-derived proteins are occasionally redeployed by the host against other viruses [64], it is possible that the bacterial versions are deployed against proteins encoded by invasive operons. This proposal is also consistent with the sporadic distribution of these families indicative of HGT and gene-loss, which is similar to that of other families of proteins implicated in providing specific selective advantage in biological conflicts $[65,66]$.

\section{General conclusions}

We present the discovery of a novel clade of ATP-grasp enzymes, the $\mathrm{R} 2 \mathrm{~K}$ clade, which includes the conserved eukaryotic protein CDC123. We show that this clade displays certain aberrant features hitherto not encountered in other members of the ATP-grasp superfamily. Nevertheless, the weight of the evidence suggests that they belong to the vast assemblage of ligases catalyzing formation of peptide bonds or similar linkages in the biosynthesis of a variety of compounds and also in the peptide-tagmodification of target proteins. We propose that the classical CDC123 family is likely to modify proteins, including possibly components of the eukaryotic eIF2 translation initiation complex. Importantly, we show that the CDC123 family had its origins in bacteria where it appears to have diversified first along with the two other families of the R2K clade. The bacterial CDC123 proteins are of two distinct types, one specifically associated with T6SS-delivered polymorphic toxin systems and the other probably functioning as effectors directed at amoeboid eukaryotic hosts. Similarly, the R2K.2 and R2K.3 families are also proposed to participate in biological conflicts, probably between bacteriophages and their hosts. Thus, our findings not only help predict an unexpected biochemical function for a poorly understood translation initiation factor but also help trace its origin back to bacterial conflict systems, where it might have been deployed as a toxin in intergenomic/interorganismal conflicts $[22,21]$.

Previously, several key components of the eukaryotic protein modification and signaling systems, such as the ADPribosyltransferases, DOT1-like protein methyltransferases, and Fic/Doc-like protein AMPylating enzymes, have been traced to polymorphic toxin- or related host targeting effector-systems of endosymbiotic bacteria $[67,18]$. CDC123 joins these as a potential protein modification system that was recruited from a bacterial effector. This observation adds one more piece of evidence to the recently proposed hypothesis that effectors from the bacterial endosymbionts of the stem eukaryotes played a fundamental role in the emergence of the characteristically eukaryotic regulatory systems and sub-cellular structures [21]. Moreover, the diversification of the R2K clade in bacteria and their phages also adds support to the hypothesis that the exchange of a common set of protein- and nucleic-acid-modifying enzymatic 
effectors between disparate bacterial conflict systems helped in their extensive diversification. Representatives of this pool of enzymes were repeatedly taken up by eukaryotes and used as components of novel regulatory systems.

\section{Methods}

Iterative sequence-profile and HMM searches were performed using the PSI-BLAST [68] and JACKHMMER web utilities (http://hmmer.janelia.org/search/jackhmmer), respectively. Queries were run against the non-redundant (nr) protein database of the National Center for Biotechnology Information (NCBI). Profile-profile comparisons were performed using the HHpred program [69]. Multiple sequence alignments were constructed using the MUSCLE alignment program [70] followed by manual adjustment as determined by high-scoring pairs detailed in homology search results and alignment with experimentallyelucidated protein structures. Alignment secondary structure predictions were performed with the JPred program [71]. Gene neighborhoods were extracted from PTT and GenBank files (downloadable from the NCBI ftp server) using Perl scripts. Sequence-based homology clustering of all proteins determined to belong to the $\mathrm{R} 2 \mathrm{~K}$ assemblage and proteins encoded in the recovered gene neighborhoods was performed with the BLASTCLUST program (http://ftp.ncbi.nih.gov/blast/documents/blastclust. html) using empirically-determined scoring and length threshold values. Visualization and manipulation of protein structure was accomplished using the PyMol program (http://www.pymol.org), structure similarity searches were performed using DaliLite [72]. Phylogenetic trees were constructed using the maximum likelihood method as implemented by the PhyML program [73].

\section{Reviewer reports}

Reviewer number 1: Dr. Michael Galperin, NCBI, NLM, NIH, United States of America

The work by Burroughs and colleagues is an important contribution that expands the diversity of the members of the ATP-grasp superfamily and proposes an enzymatic function for a widespread eukaryotic translational regulator CDC123. I have only some minor comments that might improve the presentation of the results.

1. Members the ATP-grasp superfamily are primarily ATP-dependent carboxylate-amine ligases [17], although certain members are known to function as carboxylate-thiol ligases, carboxylate-hydroxyl ligases, or phosphotransferases (kinases) [74]. It would make sense to explicitly state in the Abstract that CDC123 is predicted to function as an ATPdependent protein-peptide ligase (or a protein-amino acid ligase) and indicate that future experiments would be required to uncover the exact nature of the CDC123-catalyzed reaction.

Author response: We have made the suggested change to the abstract.

2. Sequence alignment on Figure 1 would benefit from inclusion of sequences of several ATP-grasp superfamily members of known 3D structure, such as RimK (4iwx), LysX (1uc9), glutathione synthase (1gsh), and/or biotin carboxylase (1dv1). This would help illustrate the common and distinct features of CDC123-like and typical ATP-grasp enzymes.

Author response: We agree this change increases the information conveyed by the figure; we accordingly have altered the figure and its legend according to the reviewer's recommendation.

3. While sequences of human and yeast CDC123 are indicated on Figure 1, it would make sense to explicitly list in the text the predicted active site residues of the human protein that should be targeted by future experimental efforts.

Author response: The information has been added to the text.

4. In the Figure 2 legend, the D123 label needs to be explained, and it should be stated that all other labels are specified in the main text. Since RefSeq gi numbers 159900810, 158338501, 493650113, and 492493562 are now obsolete, these need to be replaced with the respective GenBank codes. Also, gi 504297789 (Bifidobacterium longum) is repeated twice; the second instance appears to be incorrect.

Author response: We thank the reviewer for identifying these issues. We have replaced the D123 label with R2K.1 and updated the gi labels.

5. The suggested name R2K clade is hardly ideal, as this name is widely used in other contexts, such as the "R2K theology". There could be better acronyms for "RAGNYA-containing 2 lysines" (e.g., R2Lys) that would not have such connotations.

Author response: We thank the reviewer for crosschecking the R2K name and drawing this to our attention; however, given the relative obscurity of the $R 2 K$ theological doctrine within Christianity and the relative independence between the respective fields (molecular biology and theology), we believe it should not be a notable source of confusion. We admit that 
the growth of the annotated domain space makes simple yet meaningful names for domains that might not overlap with nomenclature used elsewhere increasingly difficult to assign. However, we respectfully suggest that is better to retain " $R 2 K$ ". Its primary advantage is its simplicity and it remains a proper descriptive moniker for this clade of the ATP-grasp superfamily.

\section{Reviewer number 2: Professor Sandor Pongor, International Centre for Genetic Engineering and biotechnology (ICGEB), Italy}

It is often argued that the growing body of sequence data will eventually provide answers to many important questions, eukaryogenesis remains one of the fundamental problems of evolution which is still very difficult to tackle. The main problem is the complexity of genetic and biochemical systems which have to be taken into consideration when interpreting distant structural and functional similarities. This paper presents such an interesting prediction, the authors conjecture that the eukaryotic translation initiation regulator $\mathrm{CDC} 123$ defines a novel clade of ATP-grasp enzymes which may have role in novel protein modifications. The finding is based on combining the available biochemical and genetic data on CDC123 with the inferred enzymatic function. The authors present a clear case and do not over interpret the data.

\section{Author response: We appreciate the reviewer's kind} comments.

\section{Additional file}

Additional File 1: Provides an updated tree showing the relationship of the ATP grasp modules, a more extensive alignment of the R2K clade, its phyletic distribution, and its gene neighborhoods.

\section{Abbreviations}

R2K: RAGNYA-containing lysine; DUF: Domain of Unknown Function; HMM: Hidden Markov Model; LECA: Last Eukaryotic Common Ancestor; GNAT: GCN5-like acetyltransferase; ARG: ADP-ribosylglycohydrolase.

\section{Competing interests}

The authors declare that they have no competing interests

\section{Authors' contributions}

$A M B$ and $L A$ conceived project; $A M B$ and DZ performed research and prepared figures; AMB and LA wrote the paper; All authors read and approved the final manuscript.

\section{Acknowledgements}

Research by the authors is supported by the Intramural Research Program of the National Library of Medicine, NIH, USA.

Received: 11 February 2015 Accepted: 7 April 2015

Published online: 15 May 2015

\section{References}

1. Burroughs AM, lyer $L M$, Aravind L. Structure and evolution of ubiquitin and ubiquitin-related domains. Methods Mol Biol. 2012;832:15-63. doi:10.1007/978-1-61779-474-2_2.

2. Burroughs $A M$, lyer $L M$, Aravind $L$. The natural history of ubiquitin and ubiquitin-related domains. Front Biosci (Landmark Ed). 2012;17:1433-60.

3. Iyer $L M$, Burroughs $A M$, Aravind $L$. The prokaryotic antecedents of the ubiquitin-signaling system and the early evolution of ubiquitin-like betagrasp domains. Genome Biol. 2006;7(7):R60. doi:10.1186/gb-2006-7-7-r60.

4. Burroughs AM, Ando Y, Aravind L. New perspectives on the diversification of the RNA interference system: insights from comparative genomics and small RNA sequencing. Wiley Interdiscip Rev RNA. 2014;5(2):141-81. doi:10.1002/wrna.1210.

5. Burroughs AM, Aravind L. Analysis of two domains with novel RNAprocessing activities throws light on the complex evolution of ribosomal RNA biogenesis. Front Genet. 2014;5:424. doi:10.3389/fgene.2014.00424.

6. Burroughs AM, Aravind L. A highly conserved family of domains related to the DNA-glycosylase fold helps predict multiple novel pathways for RNA modifications. RNA Biol. 2014;11(4):360-72. doi:10.4161/rna.28302.

7. Ohno K, Okuda A, Ohtsu M, Kimura G. Genetic analysis of control of proliferation in fibroblastic cells in culture. I. Isolation and characterization of mutants temperature-sensitive for proliferation or survival of untransformed diploid rat cell line 3Y1. Somat Cell Mol Genet. 1984;10(1):17-28.

8. Bieganowski P, Shilinski K, Tsichlis PN, Brenner C. Cdc123 and checkpoint forkhead associated with RING proteins control the cell cycle by controlling elF2gamma abundance. J Biol Chem. 2004;279(43):44656-66. doi:10.1074/jbc.M406151200.

9. Sanbhnani S, Yeong FM. CHFR: a key checkpoint component implicated in a wide range of cancers. CMLS. 2012;69(10):1669-87. doi:10.1007/s00018-011-0892-2.

10. Perzlmaier $A F$, Richter $F$, Seufert W. Translation initiation requires cell division cycle 123 (Cdc123) to facilitate biogenesis of the eukaryotic initiation factor 2 (elF2). J Biol Chem. 2013;288(30):21537-46. doi:10.1074/jbc.M113.472290.

11. Nissen P, Kjeldgaard M, Thirup S, Polekhina G, Reshetnikova L, Clark BF, et al. Crystal structure of the ternary complex of Phe-tRNAPhe, EF-Tu, and a GTP analog. Science. 1995;270(5241):1464-72.

12. Moller W, Schipper A, Amons R. A conserved amino acid sequence around Arg-68 of Artemia elongation factor 1 alpha is involved in the binding of guanine nucleotides and aminoacyl transfer RNAs. Biochimie. 1987;69(9):983-9.

13. Adelaide J, Finetti P, Bekhouche I, Repellini L, Geneix J, Sircoulomb F, et al. Integrated profiling of basal and luminal breast cancers. Cancer Res. 2007;67(24):11565-75. doi:10.1158/0008-5472.CAN-07-2536.

14. Zeggini E, Scott LJ, Saxena R, Voight BF, Marchini JL, Hu T, et al. Meta-analysis of genome-wide association data and large-scale replication identifies additional susceptibility loci for type 2 diabetes. Nat Genet. 2008;40(5):638-45. doi:10.1038/ng.120.

15. Soler Artigas M, Loth DW, Wain LV, Gharib SA, Obeidat M, Tang W, et al. Genome-wide association and large-scale follow up identifies 16 new loci influencing lung function. Nat Genet. 2011;43(11):1082-90. doi:10.1038/ng.941

16. Iyer LM, Abhiman S, Maxwell Burroughs A, Aravind L. Amidoligases with ATP-grasp, glutamine synthetase-like and acetyltransferase-like domains: synthesis of novel metabolites and peptide modifications of proteins. Mol Biosyst. 2009:5(12):1636-60. doi:10.1039/b917682a.

17. Galperin MY, Koonin EV. A diverse superfamily of enzymes with ATP-dependent carboxylate-amine/thiol ligase activity. Protein Sci. 1997;6(12):2639-43. doi:10.1002/pro.5560061218.

18. Zhang D, de Souza RF, Anantharaman V, lyer LM, Aravind L. Polymorphic toxin systems: Comprehensive characterization of trafficking modes, processing, mechanisms of action, immunity and ecology using comparative genomics. Biol Direct. 2012;7:18. doi:10.1186/1745-6150-7-18.

19. Zhang $D$, lyer $L M$, Aravind L. A novel immunity system for bacterial nucleic acid degrading toxins and its recruitment in various eukaryotic and DNA viral systems. Nucleic Acids Res. 2011;39(11):4532-52. doi:10.1093/nar/gkr036.

20. Zhang D, lyer LM, Burroughs AM, Aravind L. Resilience of biochemical activity in protein domains in the face of structural divergence. Curr Opin Struct Biol. 2014;26:92-103. doi:10.1016/j.sbi.2014.05.008

21. Aravind L, Anantharaman V, Zhang D, de Souza RF, lyer LM. Gene flow and biological conflict systems in the origin and evolution of eukaryotes. Front Cellular Infect Microbiol. 2012;2:89. doi:10.3389/fcimb.2012.00089.

22. Aravind L, Burroughs AM, Zhang D, lyer LM. Protein and DNA modifications: evolutionary imprints of bacterial biochemical diversification and 
geochemistry on the provenance of eukaryotic epigenetics. Cold Spring Harb Perspect Biol. 2014;6(7):a016063. doi:10.1101/cshperspect.a016063.

23. Iyer LM, Aravind L, Koonin EV. Common origin of four diverse families of large eukaryotic DNA viruses. J Virol. 2001;75(23):11720-34. doi:10.1128/JVI.75.23.11720-11734.2001.

24. Bateman A, Coggill P, Finn RD. DUFs: families in search of function. Acta Crystallogr Sect F Struct Biol Cryst Commun. 2010;66(Pt 10):1148-52. doi:10.1107/S1744309110001685.

25. Zhao G, Jin Z, Wang Y, Allewell NM, Tuchman M, Shi D. Structure and function of Escherichia coli RimK, an ATP-grasp fold, L-glutamyl ligase enzyme. Proteins. 2013;81(10):1847-54. doi:10.1002/prot.24311

26. Finn RD, Bateman A, Clements J, Coggill P, Eberhardt RY, Eddy SR, et al. Pfam: the protein families database. Nucleic Acids Res. 2014;42(Database issue):D222-30. doi:10.1093/nar/gkt1223.

27. Berman HM, Westbrook J, Feng Z, Gilliland G, Bhat TN, Weissig H, et al. The Protein Data Bank. Nucleic Acids Res. 2000;28(1):235-42.

28. Fawaz MV, Topper ME, Firestine SM. The ATP-grasp enzymes. Bioorg Chem. 2011;39(5-6):185-91. doi: 10.1016/j.bioorg.2011.08.004

29. Balaji S, Aravind L. The RAGNYA fold: a novel fold with multiple topological variants found in functionally diverse nucleic acid, nucleotide and peptide-binding proteins. Nucleic Acids Res. 2007;35(17):5658-71. doi:10.1093/nar/gkm558.

30. Denessiouk KA, Lehtonen JV, Korpela T, Johnson MS. Two "unrelated" families of ATP-dependent enzymes share extensive structural similarities about their cofactor binding sites. Protein Sci. 1998;7(5):1136-46. doi:10.1002/pro.5560070507.

31. Grishin NV. Phosphatidylinositol phosphate kinase: a link between protein kinase and glutathione synthase folds. J Mol Biol. 1999;291(2):239-47. doi:10.1006/jmbi.1999.2973

32. Tabata K, Hashimoto S. Ikeda H. ywfE in Bacillus subtilis codes for a novel enzyme, L-amino acid ligase Journal of bacteriology. 2005;187(15):5195-202. doi:10.1128/JB.187.15.5195-5202.2005.

33. Llewellyn NM, Li Y, Spencer JB. Biosynthesis of butirosin: transfer and deprotection of the unique amino acid side chain. Chem Biol. 2007;14(4):379-86. doi:10.1016/j.chembiol.2007.02.005.

34. Benson TE, Prince DB, Mutchler VT, Curry KA, Ho AM, Sarver RW, et al. X-ray crystal structure of Staphylococcus aureus FemA. Structure. 2002;10(8):1107-15

35. Liu S, Chang JS, Herberg JT, Horng MM, Tomich PK, Lin AH, et al. Allosteric inhibition of Staphylococcus aureus D-alanine:D-alanine ligase revealed by crystallographic studies. Proc Natl Acad Sci U S A. 2006;103(41):15178-83. doi:10.1073/pnas.0604905103

36. Tanabe T, Funahashi T, Nakao H, Miyoshi S, Shinoda S, Yamamoto S. Identification and characterization of genes required for biosynthesis and transport of the siderophore vibrioferrin in Vibrio parahaemolyticus. J Bacteriol. 2003;185(23):6938-49.

37. Janke C, Rogowski K, Wloga D, Regnard C, Kajava AV, Strub JM, et al. Tubulin polyglutamylase enzymes are members of the $T T L$ domain protein family. Science. 2005;308(5729):1758-62. doi:10.1126/science.1113010.

38. van Dijk J, Rogowski K, Miro J, Lacroix B, Edde B, Janke C. A targeted multienzyme mechanism for selective microtubule polyglutamylation. Mol Cell. 2007;26(3):437-48. doi:10.1016/j.molcel.2007.04.012.

39. Iyer LM, Balaji S, Koonin EV, Aravind L. Evolutionary genomics of nucleo-cytoplasmic large DNA viruses. Virus Res. 2006;117(1):156-84. doi:10.1016/j.virusres.2006.01.009.

40. Kwaik YA. Invasion of mammalian and protozoan cells by Legionella pneumophila. Subcell Biochem. 2000:33:383-410.

41. Fields BS. The molecular ecology of legionellae. Trends Microbiol. 1996:4(7):286-90

42. de Felipe KS, Pampou S, Jovanovic OS, Pericone CD, Ye SF, Kalachikov S, et al. Evidence for acquisition of Legionella type IV secretion substrates via interdomain horizontal gene transfer. J Bacteriol. 2005;187(22):7716-26. doi:10.1128/JB.187.22.7716-7726.2005.

43. Cox R, Mason-Gamer RJ, Jackson CL, Segev N. Phylogenetic analysis of Sec7-domain-containing Arf nucleotide exchangers. Mol Biol Cell. 2004;15(4):1487-505. doi:10.1091/mbc.E03-06-0443.

44. Desjardins C, Eisen JA, Nene V. New evolutionary frontiers from unusual virus genomes. Genome Biol. 2005;6(3):212. doi:10.1186/gb-2005-6-3-212.

45. Raoult D, Audic S, Robert C, Abergel C, Renesto P, Ogata H, et al. The 1.2-megabase genome sequence of Mimivirus. Science. 2004;306(5700):1344-50. doi:10.1126/ science. 1101485 .
46. Okuda A, Ohtsu M, Kimura G. Extensive degradation of mutant-type D123 protein is responsible for temperature-sensitive proliferation inhibition in 3Y1tsD123 cells. Cell Struct Funct. 1999;24(6):443-9.

47. Okuda A, Ohtsu M, Kimura G. Reversion of temperature-sensitive mutation by inhibition of proteasome-mediated degradation of mutated D123 protein Cell Struct Funct. 2001;26(4):205-14.

48. Kang WK, Icho T, Isono S, Kitakawa M, Isono K. Characterization of the gene rimK responsible for the addition of glutamic acid residues to the C-terminus of ribosomal protein S6 in Escherichia coli K12. MGG. 1989;217(2-3):281-8.

49. Schmitt T, Ogris C, Sonnhammer EL. FunCoup 3.0: database of genome-wide functional coupling networks. Nucleic Acids Res. 2014;42(1):D380-8. doi:10.1093/nar/gkt984.

50. Aravind L. Guilt by association: contextual information in genome analysis. Genome Res. 2000;10(8):1074-7.

51. Das S, Yu L, Gaitatzes C, Rogers R, Freeman J, Bienkowska J, et al. Biology's new Rosetta stone. Nature. 1997;385(6611):29-30. doi:10.1038/385029a0.

52. Iyer LM, Zhang D, Rogozin IB, Aravind L. Evolution of the deaminase fold and multiple origins of eukaryotic editing and mutagenic nucleic acid deaminases from bacterial toxin systems. Nucleic Acids Res. 2011;39(22):9473-97. doi:10.1093/nar/gkr691.

53. Basler M, Pilhofer M, Henderson GP, Jensen GJ, Mekalanos JJ. Type V secretion requires a dynamic contractile phage tail-like structure. Nature. 2012;483(7388):182-6. doi:10.1038/nature10846.

54. Nagai H, Cambronne ED, Kagan JC, Amor JC, Kahn RA, Roy CR. A C-terminal translocation signal required for Dot/lcm-dependent delivery of the Legionella RalF protein to host cells. Proc Natl Acad Sci U S A. 2005;102(3):826-31. doi:10.1073/pnas.0406239101.

55. Kubori T, Hyakutake A, Nagai H. Legionella translocates an E3 ubiquitin ligase that has multiple U-boxes with distinct functions. Mol Microbiol. 2008;67(6):1307-19. doi:10.1111/j.1365-2958.2008.06124.x.

56. Raybin D, Flavin M. Enzyme which specifically adds tyrosine to the alpha chain of tubulin. Biochemistry. 1977;16(10):2189-94.

57. Murofushi H. Purification and characterization of tubulin-tyrosine ligase from porcine brain. J Biochem. 1980;87(3):979-84.

58. Kino K, Arai T, Arimura Y. Poly-alpha-glutamic acid synthesis using a novel catalytic activity of RimK from Escherichia coli K-12. Appl Environ Microbiol. 2011;77(6):2019-25. doi:10.1128/AEM.02043-10.

59. Fitzmaurice WP, Saari LL, Lowery RG, Ludden PW, Roberts GP. Genes coding for the reversible ADP-ribosylation system of dinitrogenase reductase from Rhodospirillum rubrum. MGG. 1989;218(2):340-7.

60. Ying W, Sevigny MB, Chen Y, Swanson RA. Poly(ADP-ribose) glycohydrolase mediates oxidative and excitotoxic neuronal death. Proc Natl Acad Sci U S A. 2001;98(21):12227-32. doi:10.1073/pnas.211202598.

61. Goff CG. Chemical structure of a modification of the Escherichia coli ribonucleic acid polymerase alpha polypeptides induced by bacteriophage T4 infection. J Biol Chem. 1974;249(19):6181-90.

62. Rohrer H, Zillig W, Mailhammer R. ADP-ribosylation of DNA-dependent RNA polymerase of Escherichia coli by an NAD+: protein ADP-ribosyltransferase from bacteriophage T4. FEBS. 1975;60(1):227-38.

63. Tiemann B, Depping R, Gineikiene E, Kaliniene L, Nivinskas R, Ruger W. ModA and ModB, two ADP-ribosyltransferases encoded by bacteriophage T4: catalytic properties and mutation analysis. J Bacteriol. 2004;186(21):7262-72. doi:10.1128/JB.186.21.7262-7272.2004.

64. Snyder L. Phage-exclusion enzymes: a bonanza of biochemical and cell biology reagents? Mol Microbiol. 1995;15(3):415-20.

65. Godde JS, Bickerton A. The repetitive DNA elements called CRISPRs and their associated genes: evidence of horizontal transfer among prokaryotes. J Mol Evol. 2006;62(6):718-29. doi:10.1007/s00239-005-0223-z.

66. Burroughs AM, lyer LM, Aravind L. Two novel PIWI families: roles in inter-genomic conflicts in bacteria and Mediator-dependent modulation of transcription in eukaryotes. Biol Direct. 2013;8:13. doi:10.1186/1745-6150-8-13.

67. Aravind L, Abhiman S, lyer LM. Natural history of the eukaryotic chromatin protein methylation system. Prog Mol Biol Transl Sci. 2011;101:105-76. doi:10.1016/B978-0-12-387685-0.00004-4.

68. Schaffer AA, Aravind L, Madden TL, Shavirin S, Spouge JL, Wolf Yl, et al Improving the accuracy of PSI-BLAST protein database searches with composition-based statistics and other refinements. Nucleic Acids Res. 2001;29(14):2994-3005.

69. Soding J, Biegert A, Lupas AN. The HHpred interactive server for protein homology detection and structure prediction. Nucleic Acids Res. 2005:33 (Web Server issue):W244-8. doi:10.1093/nar/gki408. 
70. Edgar RC. MUSCLE: multiple sequence alignment with high accuracy and high throughput. Nucleic Acids Res. 2004;32(5):1792-7. doi:10.1093/nar/gkh340.

71. Cuff JA, Clamp ME, Siddiqui AS, Finlay M, Barton GJ. JPred: a consensus secondary structure prediction server. Bioinformatics. 1998;14(10):892-3.

72. Holm L, Kaariainen S, Rosenstrom P, Schenkel A. Searching protein structure databases with DaliLite v. 3. Bioinformatics. 2008;24(23):2780-1. doi:10.1093/bioinformatics/btn507.

73. Guindon S, Gascuel O. A simple, fast, and accurate algorithm to estimate large phylogenies by maximum likelihood. Syst Biol. 2003;52(5):696-704.

74. Galperin MY, Koonin EV. Divergence and convergence in enzyme evolution. J Biol Chem. 2012;287(1):21-8. 10.1074/jbc.R111.241976.

\section{Submit your next manuscript to BioMed Central} and take full advantage of:

- Convenient online submission

- Thorough peer review

- No space constraints or color figure charges

- Immediate publication on acceptance

- Inclusion in PubMed, CAS, Scopus and Google Scholar

- Research which is freely available for redistribution 